Check for updates

Cite this: RSC Adv., 2019, 9, 12001

Received 16th January 2019

Accepted 8th April 2019

DOI: $10.1039 / c 9 r a 00370 c$

rsc.li/rsc-advances

\section{Carbon nanotubes as electrophysiological building blocks for a bioactive cell scaffold through biological assembly to induce osteogenesis}

\author{
Saibo Qian, ${ }^{a}$ Zhilin Yan, ${ }^{a}$ Yongjie Xu, ${ }^{a}$ Huaping Tan, (D) *a Yong Chen, ${ }^{b}$ Zhonghua Ling ${ }^{b}$ \\ and Xiaohong Niu*c
}

Bio-functional cell scaffolds have great potential in the field of tissue regenerative medicine. In this work, a carbon nanotube (CNT) gel scaffold via specific pairing of functionalized nucleobases was developed for specifically targeted drug delivery and in vitro osteogenesis. The CNT gel scaffold with nano-fibrous architectures was established by Watson-Crick base pairing between thymine and adenine of low molecular weight heparin, respectively. As scaffold precursors, adenine and thymine functionalized heparin derivatives could additionally bind cell growth factors by the affinity interaction. The resulting nano-fibrous gel scaffolds showed excellent mechanical integrity and advanced electro-physiological functions. Potential application of the electrophysiological CNT gel scaffold in bone tissue engineering was confirmed by encapsulation of human adipose-derived stem cells (ASCs). Our results indicate that the electrically conductive networks formed by CNTs within the nano-fibrous framework are the key characteristics of cell scaffolds leading to improved ASC organization and differentiation by an extra electrical stimulus (ES). Specifically, ASCs cultured in bio-electrical gel scaffolds showed $\sim 4$ times higher spontaneous osteogenesis in combination with bone morphogenetic protein 2 (BMP-2), compared to those cultured on pristine hydrogels. This electrophysiological CNT gel scaffold containing BMP-2 exhibited beneficial effects on ASC activity and osteogenetic differentiation, which suggested a promising future for local treatment of bone regeneration.

\section{Introduction}

Injectable cell scaffolds have great potential in the field of tissue regenerative medicine, and are widely used as cell carriers and artificial tissues. ${ }^{1}$ Biodegradable hydrogels have served as highly functional cell scaffolds in addition to cell growth factors (GFs), which allow easy and homogeneous cell distribution within any defect size or shape. Although the versatility of cell scaffolds has been broadly exploited, nano-structured and biofunctional gel scaffolds are limited by conventional biopolymers and methods. There is significant interest in exploiting cell scaffolds with nano-structured architectures to construct mimics of the extracellular matrix (ECM) for cell-culture applications. In bone tissue engineering, a major issue in the use of gel scaffolds is that the establishment of a biomimetic environment is necessary for in situ osteogenesis of adipose-derived stem cells (ASCs). ${ }^{2}$

\footnotetext{
${ }^{a}$ School of Materials Science and Engineering, Nanjing University of Science and Technology, Nanjing 210094, China.E-mail: hptan@njust.edu.cn ${ }^{b}$ Department of Orthopaedics, Jinling Hospital, Nanjing 210002, China

${ }^{c}$ Department of Luoli, Nanjing Hospital of Integrated Traditional Chinese and Western Medicine, Nanjing 210014, China. E-mail: 781970731@qq.com.cn
}

Clinically, ASCs have demonstrated a broad in vitro capacity for differentiating toward cell types of all 3 germ layers, which suggests that they have wide utility in a range of regenerative strategies for multiple tissue types. Recently, undifferentiated ASCs have been seeded on a variety of cell scaffolds and examined in vivo for bone regeneration. ${ }^{3}$ ASCs are similar to bone marrow-derived stem cells (BMSCs) in that they are capable of maturing toward multiple mesodermal tissue types, including bone, cartilage and adipose; they are immunosuppressive, and they show similar surface protein marker expression. Importantly, ASCs are unique from BMSCs because they can be obtained easily in a minimally invasive manner from adipose tissue harvested through lipoplasty or liposuction in clinically applicable numbers without the need of expansion in culture. Furthermore, ASCs senesce is later than BMSCs, which may be beneficial for treating chronic or persistent conditions. For these reasons, ASCs are appealing for cell-based therapies in bone repair and regeneration.

The synthesis of a new nano-structured and bio-functional gel scaffold might therefore permit manipulation of inductive properties for osteogenesis differentiation of ASCs. Carbon nano-tubes (CNTs) have emerged as a promising nanostructured material for biomedical applications due to their unique atomic configuration, optical, mechanical and 
electronic properties, high surface area to volume ratios, as well as variable fictionalizations. ${ }^{4}$ Properly functionalized CNTs are biocompatible, non-immunogenic and photo-luminescent. These properties show potential for CNTs to be clinical nanocarriers for drug delivery and imaging. Therefore, incorporation of CNTs into biopolymer hydrogel, and potentially other biomaterials, could be useful in creating multifunctional scaffolds for both therapeutic purposes and in vitro studies. As for osteogenesis scaffolds in bone tissue engineering, most of the previous methods in the literature describing the novel functionalities are present only on the toughening modification of the CNTs for gel substrate, rather than the bio-electrical conductivity on stem cells. ${ }^{5}$

Bone formation occurs normally under the control of cells and a variety of interacting growth factors in the microenvironment. ${ }^{6}$ There is evidence that the ability of ASCs to grow and differentiate varies among physical structures and changes with bioactivities of scaffolds. Defining these variations in molecular mechanisms of osteogenesis will facilitate the development of ASCs-based therapies. Herein, we hypothesized that through hybridization, the nano-structured and bio-electrical gel scaffold can provide biochemical cues to the osteoid-like gels to generate an artificial niche for osteogenesis differentiation. It is a difficult challenge to create a bio-functional cell scaffold from ASCs to in vitro build an organized cellular construct that would permanently stimulate osteogenesis differentiation and native bone regeneration. Besides of GFs, we sought to further stimulate ASCs in CNTs-based cell scaffold by using home-designed bio-electric, in order to promote osteogenesis differentiation. Different from the existing cell scaffold technologies, we pioneered a bio-electrical cell-affinity stimulus, by which scaffold was utilized to induce osteogenesis of ASCs. Our group has employed biological assembly through specific nucleobase pairing to assemble into biodegradable and biocompatible hydrogels for GFs delivery. ${ }^{7}$ We have demonstrated that biopolymers modified with thymine and adenine functionalities spontaneously form hydrogel scaffolds via the WatsonCrick base pairing that support GFs delivery and cell encapsulation. These successful results clearly illustrate that specific nucleobase pairing promise as cell scaffolding biomaterials for tissue engineering and regenerative medicine. In this work, we present this flexible way to further assemble an electrophysiological CNTs scaffold with nano-fibrous architectures that are capable of inducing osteogenesis of ASCs. Our strategy is to use adenine and thymine functionalized heparin as hydrophilic and flexible bridges, which could synchronously bind bone morphogenetic protein (BMP-2) by specific affinity interaction.

\section{Experimental section}

\section{Materials}

Adenine, thymine, sodium heparin ( $\left.M_{\mathrm{w}} 18 \mathrm{kDa}\right)$, 1,9-dimethylmethylene blue and 3-(4,5-dimethyl-thiazol-2-yl)-2,5diphenyltetrazolium bromide (MTT) were purchased from Sigma-Aldrich. Chemical vapor deposition grown single-walled carbon nano-tubes (CNTs) were supplied by Thomas Swan \& Co. Ltd (Elicarb®). $N$-Hydroxysulfosuccinimide (sulfo-NHS) was purchased from Pierce Biotechnology Inc. (Rockford, IL). CyQuant Cell Proliferation Assay Kit, trypsin-EDTA solution, streptomycin and penicillin were purchased from Invitrogen Co. (Carlsbad, CA). Recombinant human BMP-2 (rhBMP-2) and the BMP-2 Quantikine ELISA Kit were obtained from R\&D Systems (Minneapolis, USA). All other chemicals were used as received.

\section{Synthesis of nucleotide modified biopolymers}

HP-adenine. Diethyl ether $(0.07 \mathrm{mmol})$ and $\mathrm{CHCl}_{3}(0.035$ $\mathrm{mmol})$ was added to phosphorus pentoxide $(0.035 \mathrm{mmol})$ with stirring and the mixture was heated under reflux for $12 \mathrm{~h}$ at $50{ }^{\circ} \mathrm{C}$ to get a clear solution. The solvent was then distilled off under vacuum to obtain polyphosphate ester. Adenine (0.004 $\mathrm{mmol}$ ) was dissolved in $50 \mathrm{~mL}$ of dimethylformamide with the addition of $0.25 \mathrm{~mL}$ of concentrated $\mathrm{HCl}$ with magnetic stirring. Polyphosphate ester was added to heparin $(0.0006 \mathrm{mmol})$ in $30 \mathrm{~mL}$ of dimethylformamide, followed by dropwise addition of adenine solution, and the mixture was heated to $50{ }^{\circ} \mathrm{C}$ for $24 \mathrm{~h}$. The reaction was cooled at $\mathrm{RT}$ and dimethylformamide was distilled off in vacuo. The moist residue was dissolved in water and kept in an ice-box for about $1 \mathrm{~h}$ to precipitate unreacted adenine. To the clean aqueous layer, ammonia was added to bring $\mathrm{pH}$ to 10.0 . The ammonia layer was extracted with ethyl acetate and the organic layer was evaporated. The product was dissolved in water and further purified by dialysis (MWCO 3500) against ultrapure water for 3 days. The purified solution was lyophilized to give solid foam.

HP-thymine. The clear solution of thymine $(0.004 \mathrm{mmol})$ was obtained by dissolving in $25 \mathrm{~mL}$ of $\mathrm{H}_{2} \mathrm{O}$ with the addition of $0.25 \mathrm{~mL}$ of concentrated $\mathrm{HCl}$ solution. Polyphosphate ester was added to heparin $(0.0008 \mathrm{mmol})$ in $50 \mathrm{~mL}$ of $\mathrm{H}_{2} \mathrm{O}$, followed by dropwise addition of thymine, and the mixture was heated to $50{ }^{\circ} \mathrm{C}$ for $20 \mathrm{~h}$ and cooled under ice for about $1 \mathrm{~h}$ to precipitate unreacted thymine. The acid layer is neutralized by the addition of ammonia. The ammonia layer was extracted with ethyl acetate and the organic layer is evaporated. The product was dissolved in water and further purified by dialysis (MWCO 3500) against ultrapure water for 3 days. The purified solution was lyophilized to give solid foam. For brevity, the adenine and thymine functionalized heparin are designated as HP-A and HP$\mathrm{T}$, respectively.

\section{Preparation of scaffold precursors}

Amine-functionalized CNTs $\left(\mathrm{CNT}-\mathrm{NH}_{2}\right)$ were synthesized according to an already reported procedure slightly modified. ${ }^{7}$ The loading of free amines determined by colorimetric Kaiser test was $90 \mu \mathrm{mol} \mathrm{g}^{-1}$, corresponding to $110 \mu \mathrm{mol} \mathrm{g}{ }^{-1}$ of carbon taking into account the filling yield. The $\mathrm{CNT}-\mathrm{NH}_{2}$ was also characterized by TGA performed this time under inert atmosphere, confirming the occurrence of the functionalization. Scaffold precursors were synthesized by grafting HP-A and HP-T onto CNT-NH $\mathrm{N}_{2}$, which were referred as CNT-HP-A and CNT-HP$\mathrm{T}$, respectively. To graft the nucleotide modified biopolymers onto CNT- $\mathrm{NH}_{2}, 0.5 \mathrm{~g}$ of HP-A and HP-T was dissolved in $200 \mathrm{~mL}$ nanopure $\mathrm{H}_{2} \mathrm{O}$ and incubated with EDC at $4{ }^{\circ} \mathrm{C}$ for $48 \mathrm{~h}$, 
respectively. CNT- $\mathrm{NH}_{2}$ was dissolved in nanopure $\mathrm{H}_{2} \mathrm{O}$ and added into the nucleotide modified heparin solutions under agitation with a final $\mathrm{pH}$ value of 5.6. The mixture was incubated at room temperature for $24 \mathrm{~h}$ before dialysis (MWCO 5000) for 3 days. To verify the structure of CNT-HP-A and CNTHP-T, ${ }^{1} \mathrm{H}$ NMR spectra were measured at ambient temperature using $\mathrm{D}_{2} \mathrm{O}$ as a solvent.

\section{Assembly of scaffolds}

CNT-HP-A and CNT-HP-T were dissolved in phosphate buffered saline (PBS) to form precursor solutions at a concentration of 0.5-3.0 wt\%, respectively. For the assembly of scaffold, solutions of CNT-HP-A and CNT-HP-T were thoroughly mixed with $1: 1$ ratio at room temperature by vigorous pipetting. For preparation of GFs loaded gels, BMP-2 was dissolved in the CNT-HP-A and CNT-HP-T solution in an ice bath before mixing. The final concentration of BMP-2 was fixed as $50 \mathrm{ng} \mathrm{mL}^{-1}$ (CNTs/BMP-2). To make control group, the same concentration of HP-A and HP-T in PBS was prepared. The HP-A and HP-T solutions with or without BMP-2 were thoroughly mixed by pipetting.

\section{Chemical structures}

Fourier transformed infrared (FT-IR) spectra of nucleotide functionalities of the HP-A and HP-T were measured to confirm the chemical composition. The obtained samples were recorded with FT-IR spectrometer (Nicolet Avatar 360, USA) against a blank $\mathrm{KBr}$ pellet background. The nucleotide functionalities of the HP-A and HP-T were further characterized via ${ }^{1} \mathrm{H}$ NMR (Bruker Avance). ${ }^{1} \mathrm{H}$ NMR spectroscopy results indicated that the nucleotide functionalities on HP-A and HP-T were estimated to be $57.4 \%$ and $62.3 \%$, respectively.

The grafted contents of HP-A and HP-T in the CNTs were determined quantitatively using a 1,9-dimethylmethylene blue method. ${ }^{8}$ The CNT-HP-A and CNT-HP-T were freeze-dried and digested with papain in buffer of $0.1 \mathrm{M} \mathrm{KH}_{2} \mathrm{PO}_{4}, 5 \mathrm{mM} \mathrm{Na}{ }_{2}$ EDTA and $5 \mathrm{mM}$ cysteine $\cdot \mathrm{HCl}$ at $\mathrm{pH} 6.0$ and $60{ }^{\circ} \mathrm{C}$ for $6 \mathrm{~h}$. The dye solution was prepared by dissolving $16 \mathrm{mg}$ of 1,9-dimethylmethylene blue in $1 \mathrm{~L}$ distilled water containing $3.04 \mathrm{~g}$ glycine, $2.37 \mathrm{~g} \mathrm{NaCl}$ and $95 \mathrm{~mL} \mathrm{0.1} \mathrm{M} \mathrm{HCl.} 2 \mathrm{~mL} \mathrm{1,9-dimethylmethylene}$ blue solution was added into $100 \mu \mathrm{L}$ of the papain digested solution after filtration. After $5 \mathrm{~min}$, the absorbance was measured at $525 \mathrm{~nm}$ by UV-vis spectroscopy (UV-Probe 2550, Shimadzu).

\section{Characterization of scaffolds}

Rheological experiment. Gelation kinetics of the gel scaffolds were evaluated using a commercial rheometer (AR2000, TA Instruments, New Castle, USA) with the parallel plate geometry (25 $\mathrm{mm}$ in diameter). The reaction mixture was quickly mixed and poured onto the center of the bottom plate. Standard low viscosity oil was spread at the side of the geometry to prevent water evaporation during the measurements. The evolution of storage $\left(G^{\prime}\right)$ and loss $\left(G^{\prime \prime}\right)$ moduli at a constant frequency of $0.5 \mathrm{~Hz}$ and constant strain of 0.05 was recorded as a function of time. The gelation time, as a kinetic parameter for network formation, was defined as the time of crossover of $G^{\prime}$ and $G^{\prime \prime}$.

Morphologies. Morphologies of scaffolds were characterized by utilizing SEM. For morphologies observation, the scaffolds were critical point dried (K850, Quorum, UK), and the crosssectional morphologies were viewed using a SEM instrument (JEOL, JSM-6700F) operated at $5 \mathrm{kV}$ accelerating.

Weight loss. Weight loss of initially weighed scaffolds $\left(W_{0}\right)$ was monitored as a function of incubation time in PBS at $37^{\circ} \mathrm{C}$. At specified time intervals, scaffolds were removed from the PBS and weighed $\left(W_{\mathrm{t}}\right)$. The weight loss ratio was defined as $100 \% \times$ $\left(W_{0}-W_{\mathrm{t}}\right) / W_{0}$. The weight remaining ratio was defined as 1$100 \% \times\left(W_{0}-W_{\mathrm{t}}\right) / W_{0}$.

Compressive modulus. For compressive modulus experiment, solutions described above were injected into a 24 -well culture plate for mixing to obtain columned scaffolds. Compressive modulus of elasticity was measured in the elastic region of scaffolds using a dynamic mechanical analyzer (DMA7 , PerkinElmer) in unconfined compression at a constant stress rate of $40 \mathrm{mN} \min ^{-1}$ up to $20 \%$ strain at $37{ }^{\circ} \mathrm{C}$.

Electrical characterization. The current-voltage $(I-V)$ curves were obtained at ambient temperature using a two-probe station (H19S00556, HiSOL, Japan). The currents were recorded by sweeping the voltage from 0 to $5 \mathrm{~V}$. The cables of the probe-station were connected to the inter-digitated electrodes to obtain reproducible and accurate conductivity measurements. The impedance values were measured using a CompactStat Potentiostat (CompactStat, Ivium Technologies, Netherlands) with IviumSoft software. The frequency was altered from 0.2 to $5 \mathrm{~Hz}$, and the perturbation amplitude was $25 \mathrm{mV}$.

Drug release. For BMP-2 release experiment, $100 \mu \mathrm{L}$ of scaffolds were suspended in $1 \mathrm{~mL}$ of PBS at $37{ }^{\circ} \mathrm{C}$, provided a reservoir into which BMP-2 could be released from the gel complex and subsequently measured during culture. At predetermined intervals, a $200 \mu \mathrm{L}$ sample of release medium was extracted from the sample vials and replenished with $200 \mu \mathrm{L}$ fresh release medium to maintain a constant volume. Samples were centrifuged and supernatants were collected and stored at $-20^{\circ} \mathrm{C}$ until analysis. Samples were refreshed with new PBS and vortexed after each collection. The release of BMP-2 was determined by the ELISA kit assays.

\section{Cell culture}

Human adipose-derived stem cells (ASCs) were isolated from human adipose tissue obtained from elective cosmetic surgery procedures under the institutional guideline. ${ }^{9}$ The fat tissues were minced with scissors in the collagenase solution consisted of Hanks' balanced salt solution $\left(3.0 \mathrm{~mL} \mathrm{~g}^{-1}\right.$ of fat) (SigmaAldrich, St. Louis, MO), bovine serum albumin (fatty acid free, pH 7.0, 3.5 g/100 mL Hanks') (Intergen Company, Purchase, NY) and $1 \%$ type II collagenase $\left(3.0 \mathrm{mg} \mathrm{g}^{-1}\right.$ of fat) (Worthington Biochemical Corporation, Lakewood, NJ). The centrifuge tubes were shaken at $100 \mathrm{rpm}$ for $50 \mathrm{~min}$ at $37{ }^{\circ} \mathrm{C}$. Following digestion, the content of each tube was filtered through doublelayered sterile gauze. The filtrates were then centrifuged at $1000 \mathrm{rpm}$ for $10 \mathrm{~min}$ at $37^{\circ} \mathrm{C}$, and a three layer suspension, 
consisting of a fatty layer on the top, a serum layer in the middle, and a cellular pellet at the bottom of each tube, was obtained. The fatty layer and most of the supernatant was aspirated off, leaving the pellet intact at the bottom. The pellet in each tube was then suspended in $10 \mathrm{~mL}$ of erythrocyte lysis buffer ( $\mathrm{pH} 7.4$ ), vortexed, and centrifuged again at $1000 \mathrm{rpm}$ for $10 \mathrm{~min}$ at $37{ }^{\circ} \mathrm{C}$. The pellets were suspended in the plating medium consisted of DMEM/F12 with $10 \%$ heat-inactivated fetal bovine serum, 1\% penicillin/streptomycin and 1\% Fungizone (all products obtained from Gibco, Invitrogen Corporation, Carlsbad, CA). Adherent ASCs were expanded for a period of 5-8 days at $37{ }^{\circ} \mathrm{C}$, and the medium was changed every other day until the cells achieved $80 \%$ confluence.

Cell experiments were carried out in sterilized gel scaffolds with an extra electrically stimulus (ES). ASCs were plated at a density of $5 \times 10^{6}$ cells per $\mathrm{mL}$ in 24 -well plates on samples, differentiated in 24 well inserts $0.4 \mu \mathrm{m}$ pore size. The ASCs cultured on the CNT gels were electrically stimulated at day 2 of culture using the inter-digitated electrodes under the scaffolds. The ES was applied using a waveform generator (Hioki 7075, Hioki, Japan) under a specific program (frequency $1 \mathrm{~Hz}$, voltage $3 \mathrm{~V}$, duration $10 \mathrm{~ms}$ ) for three continuous days. The electrically stimulated time of the ASCs on the scaffolds was $30 \mathrm{~min}$ in $24 \mathrm{~h}$. The medium was replenished every day during the stimulation of the scaffolds to eliminate any side effect of the generated charge. The number of ASCs encapsulated in scaffolds was quantified using the CyQuant Cell Proliferation assay. Cells were fixed and critical point dried for SEM to observe cell morphology in samples. Cytotoxicity on scaffolds was evaluated by WST1 reagent test. Cell activity and differentiation was evaluated at the end of experimental times in cell culture supernatant by Immunoenzymatic Assay kits (Invitrogen, USA) according to the manufacturer's instructions for ALP, COL-1, OCN and TGF- $\beta 1$. The cells-scaffold construct were cultured in 96 well plates in complete media and on day 7 and 14 samples were washed with PBS followed by addition of $50 \mu \mathrm{L}$ lysis solutions (0.5\% TritonX-100) in each well. Thereafter, $200 \mu \mathrm{L}$ of $1 \mathrm{mg} \mathrm{mL} \mathrm{m}^{-1}$ reaction substrate was added to each wells and incubated for $1.5 \mathrm{~h}$ at $37{ }^{\circ} \mathrm{C}$ following the manufacturer protocol. Absorbance was recorded at $405 \mathrm{~nm}$ using a spectrophotometer (ELx800, BioTek Instruments, Inc., USA). Gene expression of ALP, COL- 1 , TGF- $\beta 1$ and OSX were also evaluated by quantitative polymerase chain reaction (qPCR). After culturing in osteogenic induction media for $14 \mathrm{~d}$, ASCs under different scaffolds were treated with TRIZOL Reagent (Invitrogen) to extract the total RNA. The RNA concentration, purity and integrity were performed using a Q-5000 spectrophotometer (Quwell) at 260/280 $\mathrm{nm}$ and agarose gel electrophoresis. For cDNA synthesis, mRNA was reverse-transcribed into cDNA using the $5 \times$ PrimeScript RT Master Mix (TaKaRa) at $37^{\circ} \mathrm{C}$ for $15 \mathrm{~min}$ and $85{ }^{\circ} \mathrm{C}$ for $5 \mathrm{~s}$ according to the manufacturer's protocol. The synthesized cDNA samples were subjected to determine the expression of ALP, COL- 1 , TGF- $\beta 1$ and OSX. Gene expression was quantified by Real-Time qPCR using SYBR Green master rox (Roche Diagnostics Ltd, Mannheim, Germany) with a 7500 ABI Real-Time PCR System (Applied
Biosystems, Foster City, CA, USA). The housekeeping gene was glyceraldehyde 3-phosphate dehydrogenase (GAPDH).

\section{Statistical analysis}

The experimental data were reported as mean \pm standard deviation. Statistical analysis was performed using the two population Student's $t$-test. Statistical significance was set to $p$ value $\leq 0.05$.

This study was performed in strict accordance with the NJUST guidelines for the care and use of laboratory animals and was approved by the Institutional Animal Care and Use Committee of Nanjing University of Science and Technology (Nanjing, China).

\section{Results and discussion}

\section{Scaffold formation}

The bio-electrical CNT gel scaffold is established by the Watson-Crick base pairing between thymine (T) and adenine (A) via the hydrogen bonding (Fig. 1). For nucleobase pairing, heparin was functionalized with adenine and thymine functionalities (referred as HP-A and HP-T), respectively. HP-A and HP-T were further grafted onto the aminated CNTs as assembly precursors, respectively (referred as CNT-HP-A and CNT-HP-T). After dissolution and mixture of CNT-HP-A and CNT-HP-T in an aqueous environment, a nucleobase paired CNT gel network was assembled due to the formation of pairing complexes. Heparin is a negatively charged glycosaminoglycan (GAG), which is composed of repeated disaccharide units of alternating glucosamine and glucuronic residues heterogeneously modified by carboxyl groups and $\mathrm{N}$ - or $\mathrm{O}$-linked sulfate. Heparin has been clinically used as an anticoagulant agent. The ability of heparin to sequester and stabilize GFs due to specific affinity interaction has been exploited in the production of cell scaffolds that can mediate cell proliferation and differentiation. ${ }^{\mathbf{1 0}}$ The heparin in cell scaffold should be able to provide stabilization of GFs activity and prolonged delivery during bone regeneration. To investigate the utility and versatility of the CNT gel scaffolds for GFs delivery, the bone morphogenetic protein

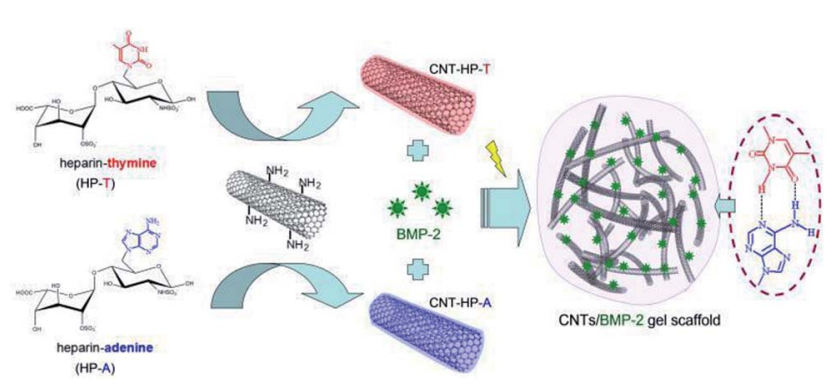

Fig. 1 Schematic assembly of CNT gel scaffold through the WatsonCrick base pairing. The scaffold precursors were designed with adenine and thymine functionalized heparin on CNTs backbone respectively, which are accessible for conjugating via specific nucleobase pairing in aqueous environment. Heparin was able to provide stabilization of BMP-2 activity and prolonged delivery via affinity interaction. 
(BMP-2) was employed as the model for affinity delivery. The BMP-2 is easily accessible to heparin, thus enabling affinity interactions in aqueous environment.

The successful modification of HP-A and HP-T was confirmed by FT-IR and ${ }^{1} \mathrm{H}$ NMR measurement. The FT-IR spectra of the biopolymers are shown in Fig. 2a. The decrease of the band at $3245 \mathrm{~cm}^{-1}$ in HP-T indicates that it undergoes a condensation reaction with the primary $-\mathrm{OH}$ group of heparin, and the appearance of a new band at $1342 \mathrm{~cm}^{-1}$ is due to the $\mathrm{C}-\mathrm{N}$ stretching vibration between heparin and thymine. In the spectrum of HP-A, a characteristic band at $3254 \mathrm{~cm}^{-1}$ and a transmittance peak at $2975 \mathrm{~cm}^{-1}$ can be assigned to the $-\mathrm{NH}$ stretching of adenine and heparin, respectively. The appearance of a new peak was at $1115 \mathrm{~cm}^{-1}$ in HP-A, which corresponds to the $\mathrm{C}-\mathrm{N}$ stretching vibration, indicating the functionalization of heparin by adenine. The FT-IR spectrum of HP-A/HP-T hydrogels was obtained with additional absorption peaks at $580 \mathrm{~cm}^{-1}$ that could be assigned to $\mathrm{H}-\mathrm{O}$ bonds of scaffold. The functionalization was also confirmed by ${ }^{1} \mathrm{H}$ NMR (Fig. 2b). As shown in spectrum of HP-T, the peak at $7.21 \mathrm{ppm}$ is due to the $-\mathrm{CH}$ protons of thymine, and the peak at $1.68 \mathrm{ppm}$ corresponds to the $-\mathrm{CH}_{3}$ protons of thymine. In spectrum of HP-A, the peaks at 8.12 and $1.85 \mathrm{ppm}$ are assigned to $-\mathrm{CH}$ and $-\mathrm{NH}_{2}$ protons of adenine, respectively.

The gelation can be controlled by the concentration of biopolymers, allowing us to fine-tune the kinetics to an application of interest. Gel scaffolds were formed via the mixing of homogeneous solutions of CNT-HP-A and CNT-HP-T in PBS. For purpose of targeted GFs delivery, BMP-2 bonded CNT gel scaffold was prepared using the same conditions, which showed an encapsulation of $50 \mathrm{ng}$ of BMP-2 per mL of gels (referred as CNTs/BMP-2 gel). Higher concentration of biopolymers mixtures was pipetted to ensure homogeneity and immediately resulted in the formation of viscoelastic gel scaffolds. For example, upon mixing with $3.0 \mathrm{wt} \%$ solutions of CNT-HP-A and CNT-HP-T, the biopolymers led to self-supporting gel scaffolds within $\sim 4$ min (Fig. 3a), which was significantly faster than the control HP-T/HP-A hydrogel (CTRL) without CNTs $(p<0.05)$. Such rapid curing kinetics is suitable for in situ tissue engineering applications. The BMP-2 integration didn't bring significant effect on gelation of the CNTs scaffold $(p>0.05)$.

Dynamic time sweep rheological experiment was conducted to monitor gel network evolution during the gelation process in
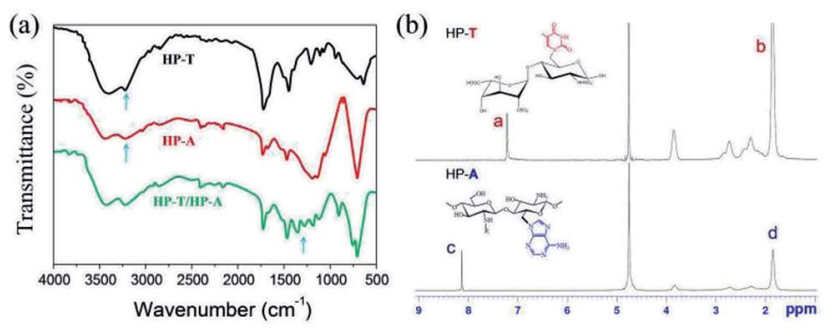

Fig. 2 (a) FT-IR spectra of thymine functionalized heparin (HP-T), adenine functionalized heparin (HP-A) and formed hydrogel. (b) ${ }^{1} \mathrm{H}$ NMR spectra of HP-T and HP-A.
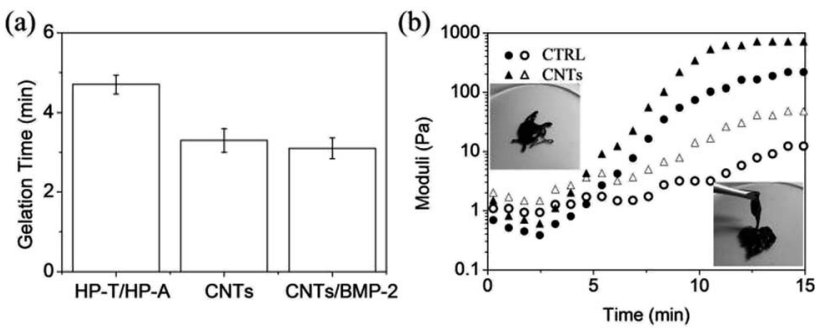

Fig. 3 (a) Gelation time of control HP-T/HP-A (CTRL), CNTs and CNTs/BMP-2 scaffolds. (b) Gelation characteristics of CTRL and CNTs as determined by in situ rheometry at $37^{\circ} \mathrm{C}$. Occurrence of a gel point as crossover between $G^{\prime}$ and $G^{\prime \prime}$. $G^{\prime}$, solid symbols; $G^{\prime \prime}$, open symbols. Concentration of BMP-2 was $50 \mathrm{ng} \mathrm{mL} \mathrm{mL}^{-1}$ of gel scaffolds. Values reported are an average $n=5, \pm$ standard deviation.

situ at $37{ }^{\circ} \mathrm{C}$ (Fig. 3b). The CNT-HP-A/CNT-HP-T mixture displayed rheological behavior that is consistent with formation of a dynamic network. Macroscopic hydrogels were obtained within less than 5 min at which the storage modulus $\left(G^{\prime}\right)$ was equal to the loss modulus $\left(G^{\prime \prime}\right)$. The data also indicate a final $G^{\prime}$ value of $\sim 100 \mathrm{~Pa}$ at time of $\sim 14 \mathrm{~min}$, signifying a structurally soft network that maintains its 3D shape with loading. The CNTs scaffold is significantly stiffer and stronger than the CTRL without CNTs $(p<0.05)$. Increasing the concentration results in increased scaffold moduli by varying 1.0 to $3.0 \mathrm{wt} \%$, which due to an increase in crosslink density. The insets in Fig. 3b demonstrate the sol-gel transition of the CNTs scaffold. Addition of CNTs does not alter the reversible/irreversible nature of the hydrogel studied, as observed by the constant angular frequency at which $G^{\prime}$ and $G^{\prime \prime}$ cross over, because the lifetime of the cross-link is unchanged. The CNT gel demonstrate the same crossover frequency as the CTRL without CNTs, even though $G^{\prime}$ plateaus at nearly $500 \mathrm{~Pa}$ with $3.0 \mathrm{wt} \% \mathrm{CNTs}$, thereby demonstrating a relatively rigid gel network with similar dynamic cross-link properties. Such rheological behavior is characteristic of soft biomaterial, hence it is suitable for injection as a cell scaffold.

\section{Scaffold characterization}

Scanning electron microscope (SEM) images characterized the morphologies of CNT gel scaffolds (Fig. 4). According to crosssectional images (Fig. 4a), the CNT gel scaffold without BMP-2
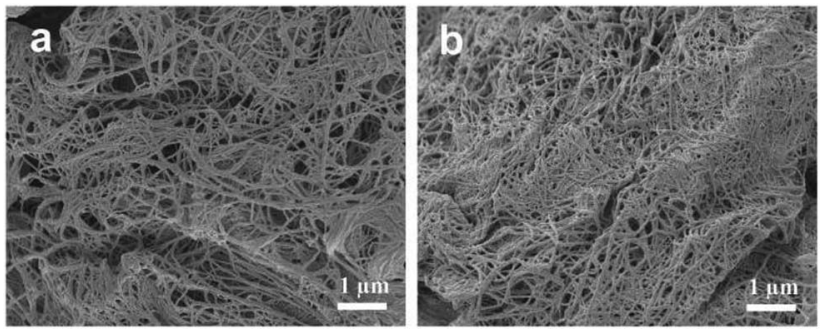

Fig. 4 SEM images characterized morphologies of (a) CNTs and (b) CNTs/BMP-2 scaffolds. Concentration of BMP-2 was $50 \mathrm{ng} \mathrm{mL}{ }^{-1}$ of gel scaffolds. 
displayed a nano-structured fibers. The BMP-2 bonded CNT gel scaffold was also observed under the same condition, which showed a similar morphology (Fig. 4b). Therefore, additional BMP-2 didn't significantly affect the morphologies of CNT gel scaffolds. These nano-fibrous architectures of gel scaffolds were attributed to the nature of CNTs, resembling self-assembly nano-fibers. The nano-fibrous CNTs/BMP-2 displayed some nano-particles than the control gel, which might contribute to conjugation of BMP-2 with heparin through the affinity interaction. The number-averaged size of nano-fibers was simultaneously determined by SEM. Size of these nano-fibers ranges from $\sim 40 \mathrm{~nm}$ to $\sim 120 \mathrm{~nm}$, and the calculated average diameter was $81.3 \mathrm{~nm}$.

The electrical conductance between the electrodes under the scaffolds was quantified to ensure accurate and reproducible conductivity measurements of the scaffolds. We observed a concentration dependent in the electrical conductivity of CNTs and CNTs/BMP-2 scaffolds. Fig. 5a shows the results of the electrical conductivity measurements at different CNTs concentration. In comparison with pristine heparin scaffold (without CNTs), the electrical conductivity was significantly enhanced upon the addition of CNTs to the scaffolds. Moreover, the scaffolds containing more CNTs had a higher conductivity compared with these low CNTs samples. For instance, the measured currents at an applied voltage of $3 \mathrm{~V}$ for the scaffolds containing the $1.0 \mathrm{wt} \%$ and $0.5 \mathrm{wt} \%$ CNTs were $22.9 \mathrm{~mA}$ and 5.1 $\mathrm{mA}$, respectively. However, the conductivity values for CNT gels did not show a significant difference except in $3.0 \mathrm{wt} \%$ CNTs concentration in which a sudden increase in the conductivity was occurred.

Compressive modulus of the CNTs and CNTs/BMP-2 gel scaffolds was studied by a dynamic mechanical analysis method (Fig. 5b). The CNTs scaffold had significantly larger compressive modulus than the control HP-A/HP-T gels $(p<0.05)$, which were 256 and $83 \mathrm{kPa}$, respectively. With addition of BMP-2 in scaffolds, the compressive modulus of the scaffolds was changed correspondingly, whereas no difference was found between them $(p>0.05)$. The data indicated a final modulus value of $\sim 264 \mathrm{kPa}$ after an addition of $50 \mathrm{ng} \mathrm{mL}{ }^{-1} \mathrm{BMP}-2$ in the CNTs scaffold, signifying a structurally robust gel network that maintains its 3D shape with loading.

Biodegradation of scaffolds is critical during bone regeneration, as it enables ASCs to invade the matrix and is also the
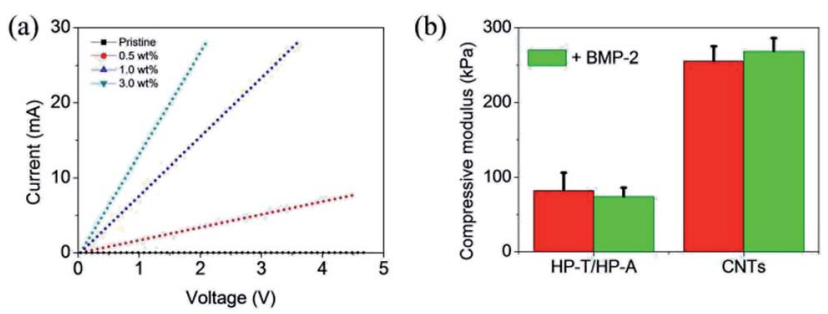

Fig. 5 (a) Current-voltage measurements of control and CNT gels as a function of concentrations. (b) Compressive modulus of control and CNTs with or without BMP-2 encapsulation at $37^{\circ} \mathrm{C}$. Values reported are an average $n=5, \pm$ standard deviation. basis for subsequent remodeling and morphogenesis to form functional bone. Therefore, weight loss of gel scaffolds was monitored as a function of incubation time in PBS at $37^{\circ} \mathrm{C}$. As shown in Fig. 6a, both of the CTRL and CNTs lost their weight steadily up to 21 days. At day 14, the weight remaining ratio of the CTRL and CNTs scaffolds were 54\% and $81 \%$, respectively. These results clearly demonstrate that the CNTs served as additional reinforcement in assembled gel scaffolds. This nanostructure influenced many of the macroscopic properties of gel scaffolds, which resulted in a decrease in the mass weight loss. It should mention that the incorporation of BMP-2 also didn't bring a significant influence on weight loss of both gel scaffolds.

Controlled release of BMP-2 can be achieved through their anchorage at predetermined locales of heparin in scaffolds. Strikingly, over a initial period of 21 days, less than $25 \%$ of encapsulated BMP-2 was released from the CNT gel scaffold, which showed significantly slower rate compared to the control group $(p<0.05)$ (Fig. 6b). We speculate that this delivery system relied on the active inclusion of heparin in CNT gel scaffold via affinity interaction with BMP-2, as well as the stable nanostructure. The BMP-2 slowly diffused out of the CNT gel scaffold, indicating that it was capable of prolonged delivery.

\section{In vitro osteogenesis}

It is envisioned that our gel scaffold would not only house stem cells, but also provide osteo-inductive cues in a dynamic functional system that enables cell-induced rearrangement of collagenous matrix and its mineralization. Through this, a microenvironment is enabled to sustain and accommodate osteoblastic differentiation of ASCs homogenously seeded within the scaffolds. To test the hypothesis, human ASCs were seeded within CNTs scaffolds containing BMP-2, and their osteogenesis differentiation and mediated mineralization were compared to those seeded in gel scaffolds alone. The availability of gel scaffolds was allowed to verify that the electrically stimulus (ES) have beneficial effects on the osteoblast proliferation and differentiation.

For bone tissue engineering applications, biomaterials scaffolds often used for loading cells, and to enhance osteogenesis of tissue engineered bone. The transplantation of BMP2 in biomaterial scaffolds has been studied, since scaffolds with BMP-2 could result in the increase of osteogenic response.
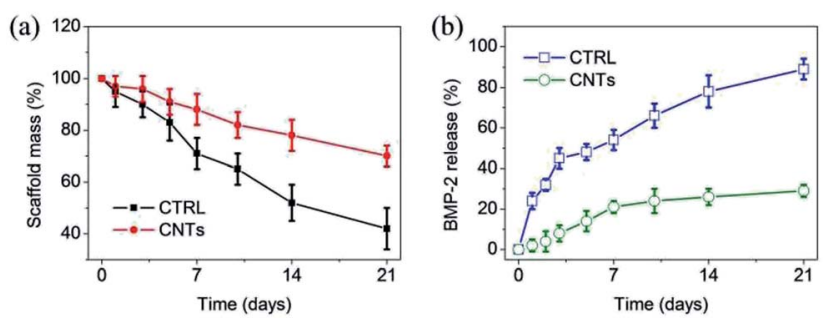

Fig. 6 (a) Degradation of control and CNT gels with respect to weight loss in PBS at $37^{\circ} \mathrm{C}$. (b) Cumulative release profiles of BMP-2 from control and CNT gels as a function of time in PBS at $37^{\circ} \mathrm{C}$. Concentration of BMP-2 was $50 \mathrm{ng} \mathrm{mL}^{-1}$ of gel scaffolds. Values reported are an average $n=5, \pm$ standard deviation. 

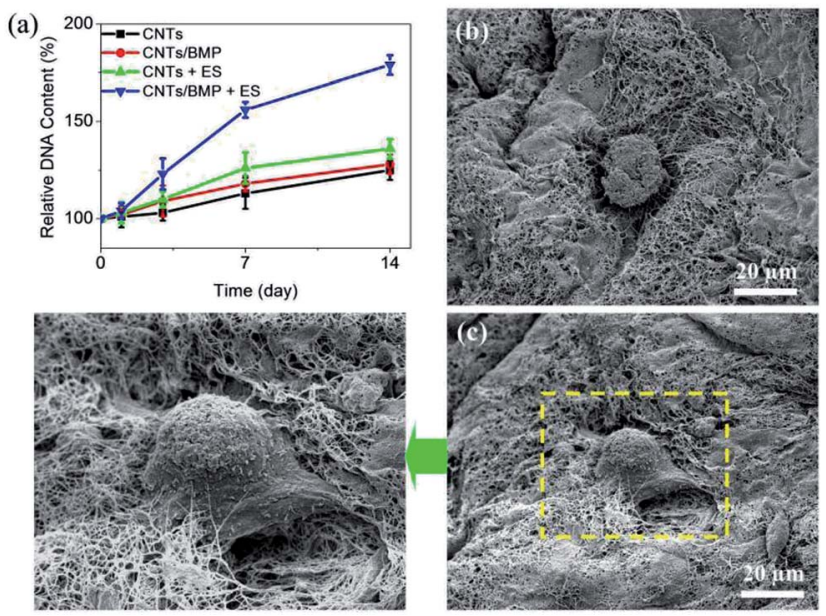

Fig. 7 Proliferation of ASCs in scaffolds by electrically stimulus (ES). (a) DNA contents of encapsulated ASCs in CNTs scaffolds as a function of culture time. (b and c) SEM images showed ASCs and BMP-2 containing CNTs matrices after 7 and 14 days of culture. Cell seeding density: $5 \times 10^{6}$ cell per $\mathrm{mL}$ gel. Values reported are an average $n=5$, \pm standard deviation.

Fig. 7a shows the time course of change in relative DNA content of the cells in the CNTs and CNTs/BMP-2 scaffolds. Basically, DNA content in the scaffolds progressively increased after encapsulation compared with initial DNA content, which was further significantly increased after 3 days of culture $(p<0.05)$. As for the ES on cells, there was no significant change of DNA number in the initial 2 days of culture $(p>0.05)$, but its DNA content was significantly increased after 3 days culture $(p<$ 0.05). The ASCs residing in the CNTs/BMP-2 scaffolds were observed by SEM after 7 and 14 days incubation (Fig. $7 \mathrm{~b}$ and c). Roundly shaped cells were distributed in the scaffold after 7 days, indicative of the highly bioactive nature of the CNTs (Fig. 7b). Long incubation time was performed in order to probe a range of differentiative responses of the scaffold for ASCs. As shown in Fig. 7c, after 14 days incubation, the encapsulated ASCs in CNTs/BMP-2 showed polygonous morphologies compare to those on day 7. As the importance evidence for ASCs differentiation, SEM image also showed that lots of collagenlike fibrils accumulated on the cells. These results demonstrated that the multifunctional CNTs/BMP-2 was bioactive and preserved the proliferation and differentiation of the encapsulated ASCs, especially by the ES. The electrically stimulus mediated BMP-2 loaded scaffold induced differentiation of ASCs, and coupled with the cell proliferation data (Fig. 7a), suggested a novel potential mechanism for targeted osteogenesis of stem cells.

Recently, CNTs and graphene have attracted significant attention in myocardial tissue engineering applications arising from their outstanding electrical and mechanical properties. ${ }^{5}$ Incorporating CNTs and graphene into cell scaffolds leads to enhanced scaffold flexibility, strength, and electrical conductivity. Although some electrical scaffolds based on CNTs and graphene are quite successful for myocardial tissue engineering, the biomedical applications of these scaffolds are strongly limited by the intrinsic inability to translate ASCs into bone tissue engineering applications. There is a clinical need for development of bioactive gel scaffold that approximate the osteogenesis of ASCs for bone regeneration. In our study, ASCs were cultured up to 14 days by considering the mean time required for the detection of in vitro osteoblastic markers production. The BMP-2 deeply influenced cultured cells in gel scaffolds, affecting osteoblast activities. As shown in Fig. 8a, cells grown on BMP-2 containing scaffolds showed an increased metabolic activity. In comparison to controls, BMP-2 and electrically stimulus enhanced Alkaline Phosphatase (ALP) production $(p<0.05)$ (Fig. $8 \mathrm{~b})$, which is an early marker of differentiation. In particular, the higher values of type I ProCollagen (COL-1) and Osteocalcin (OCN) observed on CNTs/ BMP-2 scaffolds at both 7 and 14 days (Fig. 8c and d), indicated a significant stimulation of cell to an early differentiation and deposition of ECM by the electrically stimulus.

Qualitatively, a higher mineral content was observable in ASCs seeded the CNTs and CNTs/BMP-2 scaffolds after ES incubation, when compared to the control gel. The promotion of CNTs mediated ES to ASCs osteogenic differentiation was assessed by quantitative polymerase chain reaction (qPCR). Four osteogenic differentiation markers at the RNA level were measured. ALP can regulate numerous genes associated with osteogenic differentiation as a key transcription factor in early period, and usually is treated as landmark gene. COL-1 and Osterix (OSX) are the most important two types of representation form in osteogenic differentiation maturation period, and have important effect on osteogenesis. The results of qPCR analysis were in agreement with the significant activation of ALP, COL-1 and OSX expression (Fig. 9a-c). OSX is a gene that specifically regulates osteoblast maturation and differentiation, while transforming growth factor $\beta 1$ (TGF- $\beta 1$ ) is another important regulator in the bone remodeling network, modulating the interplay between osteoblast and osteoclast in the bone formation-resorption balance. Our results showed that the
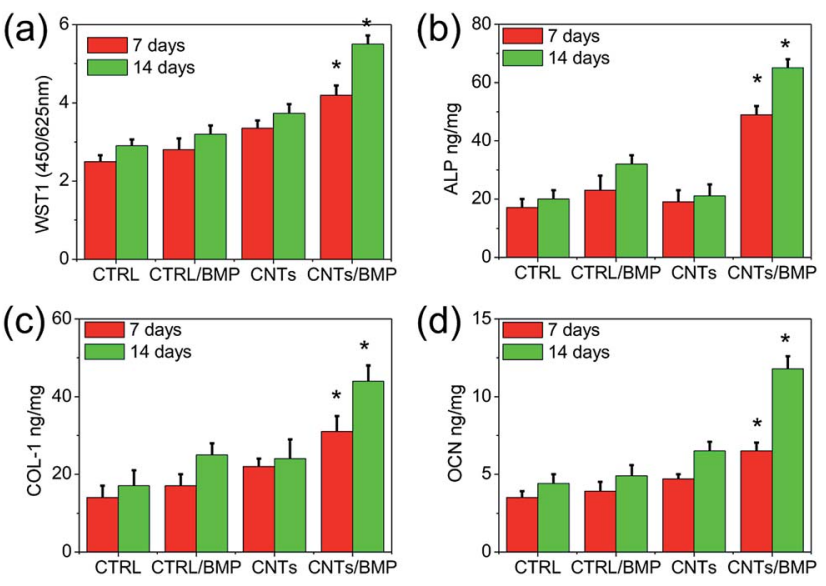

Fig. 8 ASCs viability (WST1) and activities (ALP, COL-1, OCN) after 7 and 14 days of electrical culture in control and CNTs scaffolds. Concentration of BMP- 2 was $50 \mathrm{ng} \mathrm{mL}^{-1}$ of gel scaffolds. Cell seeding density: $5 \times 10^{6}$ cell per $\mathrm{mL}$ gel. Values reported are an average $n=5$, \pm standard deviation. Statistical significance is denoted by $*(p \leq 0.05)$. 

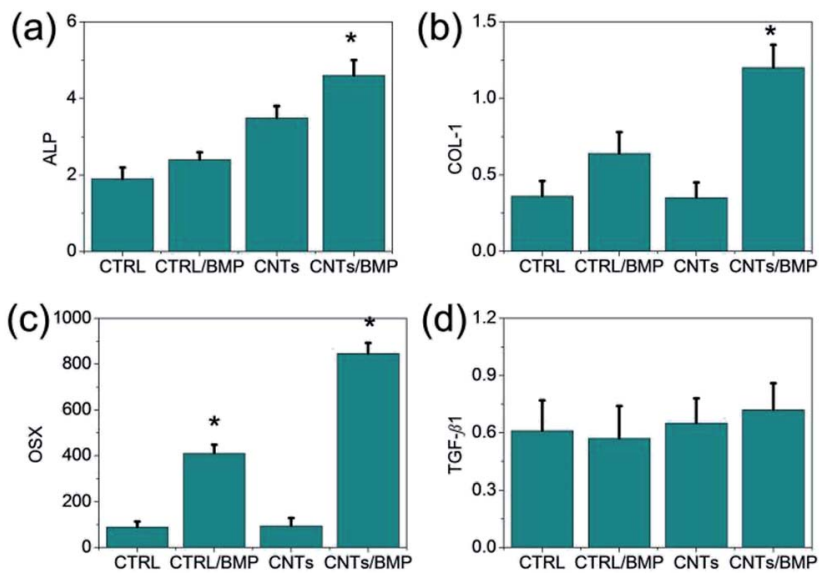

Fig. 9 Gene expression of cell growth and osteoblastic phenotype related genes (ALP, COL-1, OSX and TGF- $\beta 1$ ) during in vitro culture after 14 days of electrical culture. Expression level was normalized with respect to the control on day 14 . Cell seeding density: $5 \times 10^{6} \mathrm{~mL}^{-1}$ gel. Statistical significance is denoted by $*(p \leq 0.05)$.

effects of nano-structures and BMP-2 on cell didn't significantly affect TGF- $\beta 1$ expression on day 14 incubation, as well as the ES culture (Fig. 9d). In comparison with control heparin scaffolds, the CNTs and CNTs/BMP-2 didn't show higher TGF- $\beta 1$ gene expression after 14 days culture, as well as low levels of TGF- $\beta 1$ activity in the culture supernatants. The data on cell activities are consistent with these results, since they show that low levels of TGF- $\beta 1$ correspond to reduced proliferation rates from day 7 to day 14 (Fig. 7a), related to enhancement of osteoblastic differentiation. These results demonstrated that the ASCs were similar to BMSCs in that they were capable of maturing toward bone, which showed similar surface protein marker expression. In combination with electrical stimulate, this electrophysiological CNTs scaffold containing BMP-2 exhibited beneficial effects on ASCs activities and osteogenesis differentiation, which suggested a promising future for bone regeneration.

\section{Conclusions}

We have developed a novelly electrophysiological CNT gel scaffold that specifically allows for targeted BMP-2 delivery and osteogenesis of ASCs. The CNT gel scaffold was attributed to the mechanism of the Watson-Crick base pairing via intermolecular hydrogen bonding between thymine and adenine. We demonstrated that the addition of CNTs into biopolymer led to a hybrid scaffold with significantly altered electrophysiological and mechanical properties. Compression modulus and electrical conductivity of the scaffolds, as well as key indicators of their biocompatibility, showed clear dependence on CNTs concentration. CNTs formed electrically conductive and collagen fibril-like nano-fibers, which mechanically strengthened the scaffold, promoted ASCs proliferation and improved osteogenesis. In combination with electrical stimulate, scaffolds fabricated by seeding ASCs onto CNT gels showed strong spontaneous and stimulated synchronous osteogenesis. This electrophysiological gel scaffolds could be used for stem cells to create bone constructs with improved organization, electroactivity and mechanical integrity.

\section{Conflicts of interest}

There are no conflicts to declare.

\section{Acknowledgements}

This study is financially supported by the National Undergraduate Training Program for Innovation and Entrepreneurship (201710288070), the Natural Science Foundation of Jiangsu Province (BK20171113) and the Training Project for Leading Talents of Jiangsu Province Traditional Chinese Medicine (LJ200921).

\section{Notes and references}

1 (a) M. Morishita, A. M. Lowman, K. Takayama, T. Nagai and N. A. Peppas, J. Controlled Release, 2002, 81, 25; (b) Y. Cheng, H. Yang, Y. Yang, J. Huang, K. Wu, Z. Chen, X. Wang, C. Lin and Y. Lai, J. Mater. Chem. B, 2018, 6, 1862; (c) S. M. Damaraju, Y. Shen, E. Elele, B. Khusid, A. Eshghinejad, J. Li, M. Jaffe and T. L. Arinzeh, Biomaterials, 2017, 149, 51; (d) B. Tandon, J. J. Blaker and S. H. Cartmell, Acta Biomater., 2018, 73, 1; (e) H. Tan, M. Fan, Y. Ma, J. Qiu, X. Li and J. Yan, Adv. Healthcare Mater., 2014, 3, 1769.

2 (a) D. K. Yi, S. S. Nanda, K. Kim and S. T. Selvan, J. Mater. Chem. B, 2017, 5, 9429; (b) H. Chen, X. Xing, Y. Jia, J. Mao, Z. Zhang and H. Tan, J. Nanosci. Nanotechnol., 2016, 16, 5562; (c) M. H. Hettiaratchi, R. E. Guldberg and T. C. McDevitt, J. Mater. Chem. B, 2016, 4, 3464; (d) H. Tan, X. Gao, J. Sun, C. Xiao and X. Hu, Chem. Commun., 2013, 49, 11554; (e) M. Fan, Y. Ma, J. Mao, Z. Zhang and H. Tan, Acta Biomater., 2015, 20, 60.

3 (a) D. A. Bourne, R. D. Thomas, J. Bliley, G. Haas, A. Wyse, A. Donnenberg, V. S. Donnenberg, I. Chow, R. Cooper, S. Coleman, K. Marra, P. F. Pasquina and J. P. Rubin, Plast. Reconstr. Surg., 2018, 142, 1349; (b) L. E. Kokai, K. G. Marra and E. E. Kershaw, Endocrinology, 2015, 156, 4375; (c) D. M. Minteer, K. G. Marra and J. P. Rubin, Clin. Plast. Surg., 2015, 42, 169; (d) L. E. Kokai, K. Marra and J. P. Rubin, Transl. Res., 2014, 163, 399.

4 (a) M. K. Khan, J. Luo, Z. Wang, R. Khan, X. Chen and Y. Wan, J. Mater. Chem. B, 2018, 6, 1640; (b) B. Ma, F. Liu, S. Zhang, J. Duan, Y. Kong, Z. Li, D. Tang, W. Wang, S. Ge, W. Tang and H. Liu, J. Mater. Chem. B, 2018, 6, 6459; (c) A. P. H. J. Schenning and E. W. Meijer, Chem. Commun., 2005, 41, 3245; (d) S. Samavedi, A. R. Whittington and A. S. Goldstein, Acta Biomater., 2013, 9, 8037.

5 (a) C. Spinato, A. P. R. de Garibay, M. Kierkowicz, E. Pach, M. Martincic, R. Klippstein, M. Bourgognon, J. T. Wang, C. Ménard-Moyon, K. T. Al-Jamal, B. Ballesteros, G. Tobias and A. Bianco, Nanoscale, 2016, 8, 12626; (b) E. Heister, E. W. Brunner, G. R. Dieckmann, I. Jurewicz and A. B. Dalton, ACS Appl. Mater. Interfaces, 2013, 5, 1870; (c) 
S. Ahadian, S. Yamada, J. Ramón-Azcón, M. Estili, X. Liang, K. Nakajima, H. Shiku, A. Khademhosseini and T. Matsue, Acta Biomater., 2016, 31, 134; (d) S. R. Shin, S. M. Jung, M. Zalabany, K. Kim, P. Zorlutuna, S. bok Kim, M. Nikkhah, M. Khabiry, M. Azize, J. Kong, K. Wan, T. Palacios, M. R. Dokmeci, H. Bae, X. Tang and A. Khademhossein, ACS Nano, 2013, 7, 2369.

6 (a) Y. Song, Y. Zhou and L. Chen, J. Mater. Chem., 2012, 22, 2512; (b) Y. Song, H. Wang, X. Zeng, Y. Sun, X. Zhang, J. Zhou and L. Zhang, Bioconjugate Chem., 2010, 21, 1271.

7 (a) M. Fan, J. Yan, H. Tan, Y. Miao and X. Hu, J. Mater. Chem. B, 2014, 2, 8399; (b) H. Tan, C. Xiao, J. Sun, D. Xiong and X. Hu, Chem. Commun., 2012, 48, 10289; (c) M. Fan, J. Yan, H. Tan, D. Ben, Q. He, Z. Huang and X. Hu, Macromol. Biosci., 2014, 14, 1521.

8 (a) H. Tan, J. Wu, D. Huang and C. Gao, Macromol. Biosci., 2010, 10, 156; (b) H. Tan, C. R. Chu, K. A. Payne and
K. G. Marra, Biomaterials, 2009, 30, 2499; (c) H. Tan, H. Li, J. P. Rubin and K. G. Marra, J. Tissue Eng. Regener. Med., 2011, 5, 790.

9 (a) H. Tan, C. M. Ramirez, N. Miljkovic, H. Li, J. P. Rubin and K. G. Marra, Biomaterials, 2009, 30, 6844; (b) H. Tan, Q. Shen, X. Jia, Z. Yuan and D. Xiong, Macromol. Rapid Commun., 2012, 33, 2015; (c) H. Tan, J. P. Rubin and K. G. Marra, Macromol. Rapid Commun., 2011, 32, 905.

10 (a) X. Xu, A. K. Jha, R. L. Duncan and X. Jia, Acta Biomater., 2011, 7, 3050; (b) N. Chinen, M. Tanihara, M. Nakagawa, K. Shinozaki, E. Yamamoto, Y. Mizushima and Y. Suzuki, J. Biomed. Mater. Res., Part A, 2003, 67, 61; (c) H. Tan, L. Lao, J. Wu, Y. Gong and C. Gao, Polym. Adv. Technol., 2008, 19, 15; (d) H. Tan, Q. Zhou, H. Qi, D. Zhu, X. Ma and D. Xiong, Macromol. Biosci., 2012, 12, 621. 